\title{
Patterns in cognitive phenomena and pluralism of explanatory styles
}

Angela Potochnik and Guilherme Sanches de Oliveira

\begin{abstract}
Debate about cognitive science explanations has been formulated in terms of identifying the proper level(s) of explanation. Views range from reductionist, favoring only neuroscience explanations, to mechanist, favoring the integration of multiple levels, to pluralist, favoring the preservation of even the most general, high-level explanations, such as those provided by embodied or dynamical approaches. In this paper, we challenge this framing. We suggest that these are not different levels of explanation at all but, rather, different styles of explanation that capture different, cross-cutting patterns in cognitive phenomena. Which pattern is explanatory depends on both the cognitive phenomenon under investigation and the research interests occasioning the explanation. This reframing changes how we should answer the basic questions of which cognitive science approaches explain and how these explanations relate to one another. On this view, we should expect different approaches to offer independent explanations in terms of their different focal patterns and the value of those explanations to partly derive from the broad patterns they feature.
\end{abstract}

Cognitive science is a big tent, housing a variety of scientific disciplines relevant to the study of cognition. As tends to happen in big tents, there are disputes about who has priority and who may not belong at all. In particular, there is regular debate about the explanatory value of the various scientific disciplines comprising the cognitive sciences. At root, the questions are: which cognitive science approaches explain cognitive phenomena, and how do these explanations relate to one another?

This debate is often put in terms of identifying the proper level(s) of explanation. There is an intuitive sense in which, say, neuroscience targets entities located at a lower level than does 
ecological psychology. Neuroscience investigates phenomena occurring within the nervous system, whereas ecological psychology investigates how whole organisms interact with their social and environmental surroundings. The former thus seems to focus on entities that are proper parts of-and thus at a lower level than-the systems investigated by the latter. The debate about levels of explanation in cognitive science regards which level or levels explain cognitive phenomena, and thus which disciplines in cognitive science generate explanations. Explanatory reductionists assert the explanatory priority of the neurological components of cognitive systems. Others advocate explanatory integration, the view that successful cognitive science explanations must draw from multiple fields to address multiple levels. Finally, explanatory pluralists hold that different cognitive phenomena are best explained at different levels, and so by different fields.

In this paper, we challenge this framing of the debate about explanation in the cognitive sciences. Interpreting this as a question of levels of explanation is, we argue, misleading in a way that has confused the debate. In Section 1, we characterize the debate over levels of explanation. In Section 2, we argue that formulating questions about cognitive science explanations in terms of levels misconstrues the relationship among candidate cognitive science explanations. In Section 3, we motivate a different form of explanatory pluralism that we call pluralism of explanatory styles, according to which there are a variety of cross-cutting styles of cognitive science explanation, capturing different patterns and responding to different research interests. Finally, in Section 4, we outline the implications of this view for explanatory practices in the cognitive sciences.

\section{The Debate About Levels of Explanation}

Across the sciences and philosophy of science, the terminology of "levels" has often been used to distinguish among scientific approaches---those that focus on the relatively large versus the miniscule, those that emphasize components versus integrated wholes, those that dig into the 
details versus abstract away from the details. These invocations of levels have led to the question of the proper level or levels for scientific explanations, and this question is especially apt in cognitive science, an interdisciplinary endeavor that quite obviously includes study of multiple levels.

The concept of levels has been invoked in a number of different ways in science and philosophy of science, and these uses can seem similar, but differ in their details (raver, 2007; Potochnik, 2017). In cognitive science, Marr's (1982) idea that the brain implements processes described at higher "algorithmic" and "computational" levels has been tremendously influential; this notion of levels regards specificity or generality of description. A mechanistic conception of levels is similarly influential in cognitive science; this is the idea that levels are ordered according to the components and subcomponents of mechanisms. This is akin to a compositional conception of levels in terms of parts and wholes, though even these closely related levels concepts differ in their details (Eronon, 2013).

Commonly described levels in cognitive science include molecular, neurological, representational/computational, and social/cultural. These seem to blend together specificity of description (e.g. algorithmic modeling) with size ordering (e.g. molecular versus neurological versus entire organism-environment relationships). The debate about levels of explanation plays out in this context. The options seem to include (1) privileging one level of explanation, as exemplified by reductionism; (2) requiring multiple levels for explanation, as with explanatory integration; and (3) admitting multiple different levels as separately explanatory, as with explanatory pluralism. Each of these positions has been advocated in cognitive science.

According to explanatory reductionism, in order to explain phenomena, scientists should look to the component parts of systems, and then to ever-smaller subcomponents. In cognitive science, this typically means anticipating neurological or even molecular explanations for all cognitive phenomena. For example, Kandel et al. (2013) claim that "all behavior is an expression of neural activity" (xlii) and that this is the source of "the biological principles that 
underlie human behavior" (18). This suggests that all cognitive science explanations are ultimately grounded in information about neural activity. If neural activity is truly the basis for "the biological principles that underlie human behavior," then it seems cognitive psychology, ecological psychology, and all the rest offer nothing explanatory that neuroscience is not better suited to provide.

There are disagreements about precisely how the neural basis of cognition is best investigated and understood---or how far the reduction should go. Functional neuroimaging focuses on the activity of neuron populations and brain areas, which is relatively coarse-grained compared to approaches that focus on the molecular and cellular basis of cognition. John Bickle endorses a radical version of this view he calls 'ruthless reductionism' (Bickle, this issue; Bickle, 2003.) In his view, not only must we turn to neural activity, we must go "further down' to cellular, synaptic, and ultimately molecular biological mechanisms" (Bickle 2003, p. 4). Bickle describes cognitive psychology and even cognitive neuroscience as "higher levels" that are of merely heuristic value: they are useful only to the extent that they help us "in the search for lower level neuronal mechanisms" (p. 130). In a vivid illustration of the reductionist ideal, Bickle suggests that poverty can only explain criminal behavior if poverty affects neuronal ion exchange: "if, e.g., poverty causally influences behavior, it must be "transducible" down to this level of biochemical mechanism" (p. 60). This is a particularly extreme version of the broader reductionist idea that neural activity holds the key to all explanation in the cognitive sciences. From the perspective of cognitive science's big tent, any approach that centers neural activity in this way is a version of brain-centered reductionism.

In contrast to reductionism, mechanists emphasize the need for integration among the explanatory insights gleaned from different fields of cognitive science to explain cognitive phenomena. Milkowski et al. (2018), for example, argue that cognitive science is in the business of "building integrated explanations of the mechanisms involved [in cognition]," explanations that must draw from a variety of disciplinary perspectives. Whereas reductionists tout the 
explanatory value of "lower level" neural or even molecular goings-on, mechanists and others who advocate explanatory integration emphasize the need for explanations to draw from multiple levels. Paul Thagard says:

I expect that computational models will increasingly aid our understanding of the relations between different levels of mechanisms-for example, helping to relate the social to the cognitive, the cognitive to the neural, and the neural to the molecular $(2005$, p. 218-219).

On this view, no discipline has sole explanatory advantage, but rather each provides insight into a distinct level of cognitive systems, so they must be integrated to achieve full understanding. An integrative stance about cognitive science explanation is also embraced by some who do not share this emphasis on mechanism. Gallagher, et al. (2015), for instance, emphasize the aim of "multi-scale explanations involving factors at various scales (neuroscientific, psychological, phenomenological, social, and so on) all contributing to an integrated explanation" (p. 156-157).

The positions of explanatory integration and explanatory reductionism seem to agree that successful explanations include neural and even molecular details. Explanatory pluralists disagree. Many varieties of pluralism about science have been developed. Pluralists about explanation typically construe the variety of explanatory insights from different scientific investigations as a permanent, desirable feature of science. This too has been justified by appeal to levels; for example, Kitcher (1984) argues that the existence of multiple explanations is "not simply... a temporary feature of our science stemming from our cognitive imperfections but... the reflection of levels of organization in nature" (p. 371). In cognitive science, explanatory pluralism amounts to the view that separate explanations may be developed that cite distinct levels of phenomena, e.g. molecular, neural, cognitive, and social, and that these explanations are independent from one another. Here's an example of explicit advocacy for explanatory pluralism with regards to levels: 
It is plausible to think that there are as many levels of description available as there are levels of organization... We may describe humans in terms of their physical make-up, their chemical constitution, their physiological structure, their gross anatomy, their cognitive capacities, their social role, and much more. All these give a distinctive perspective on human psychology (Richardson 2009, p. 474, emphasis added). For the explanatory pluralist, each of these perspectives may be able to independently explain some cognitive phenomena without recourse to explanatory resources from other levels.

Advocates of embodied, ecological, and dynamical approaches in cognitive science often endorse explanatory pluralism. This supports the view that these approaches can be genuinely explanatory, even though they are not straightforwardly integratable with or reducible to explanations at the neural level. For example, Dale et al. advocate the explanatory autonomy of dynamical systems theory as follows:

We use many theories to understand the universe. This basic idea, often called explanatory pluralism, derives from the diverse levels of organization in the universe, and the equally diverse explanatory goals of human beings-concluding that many and multifarious theories are needed (2009, p. 739).

These authors grant that some circumstances call for explanatory integration. Still, they (and other explanatory pluralists) uphold the possibility of independent explanations, and many explicitly justify this with reference to different levels of organization. ${ }^{1}$ Notice that explanatory pluralism is the only view we've surveyed on which neural information is not necessary for all cognitive science explanations.

This is the lay of the land as we see it for views about levels of cognitive science explanation. Explanatory pluralists emphasize the possibility of explaining cognitive phenomena

\footnotetext{
${ }^{1}$ To be clear, Dale et al. (2009) do not frame the motivation for pluralism as deriving solely from level of organization but also as due to the diversity of explanatory goals scientists have. We agree with them on the latter, as will become clear below.
} 
in different ways at different levels. Integrationists emphasize the need for explanations to draw from multiple levels, including neural and even molecular levels, to fully explain. Reductionists hold that neural or even molecular investigations are (or, with research advances, will be) uniquely poised to explain all cognitive phenomena. All three positions tend to share the presumption that the different phenomena involved in cognition, and the different investigations that target them, hold a hierarchical or leveled relationship to one another. In what follows, we challenge this presumption.

\section{Explanations Don't Come in Levels}

Above we noted that there are different concepts of levels, related (at least) to degree of abstraction, material composition, and mechanistic composition, and these can all be bound up with the question of level of explanation. Another levels concept at play in debates about explanation relates to the realization, or implementation of one property by others; this is somewhat related both to degree of abstraction and mechanistic composition, but it does not straightforwardly correspond to either. These different concepts that can be used to articulate levels are sometimes aligned, but often not (Potochnik, 2017). But, regardless of which concept or concepts are used to articulate levels, in our view, the entities and properties that feature in different "levels" of explanation in cognitive science fail to relate to one another in any of the anticipated ways. It's not simply that cognitive science explanations sometimes do not perfectly fit leveling schemes; we believe "levels" quite often misdescribes the relationship among different approaches to cognitive science explanation. Simply put, different approaches to cognitive science explanations are not arranged into levels.

Let's return to the example of criminal behavior introduced above in the context of Bickle's ruthless reductionism. Neuroscientists who investigate criminal behavior study happenings in the brain that may relate to such behavior. But the biochemical mechanisms that Bickle emphasizes are hard to come by, as very many things happen in the brain, and "we have 
no prior knowledge of what the relevant level of brain organization is for any given behavior" (Krakauer et al., 2017, p. 480). So, instead, neuroscientists often focus on activity in entire brain regions. In forensic neuroimaging, for example, researchers look for neural biomarkers of aggressive and impulsive behavior (Bufkin and Luttrell, 2005; Aharoni et al., 2013). Personality and cognitive psychologists, in turn, have studied criminal behavior by focusing on, for example, cognitive processing patterns associated with antisocial personality disorder (Riser and Kosson, 2013), personality characteristics such as rejection sensitivity and proneness to rumination (Bartolo et al., 2010), and impaired social information-processing skills (Bennet et al., 2005). Some psychological research focuses on the situational, social and environmental factors associated with crime incidence (see, e.g., Clarke, 1980; Brown and Altman, 1983; Perkins et al., 1993; Kuo and Sullivan, 2001; Donovan and Prestemon, 2012). Embodied and ecological approaches have in turn focused on the perceptual patterns of offenders (McArthur and Baron, 1983; Topalli, 2005; Topalli et al., 2015, Renaud et al., 2013) and the dynamics of social coordination in groups (Coleman et al., 2007; Tesser and Achee, 1994; Barrett et al., 2012).

Each of these approaches potentially provides explanatory insight into criminal behavior: variously, the role of neural activity, psychological disorders, social abilities, environmental factors, and perceptual and social dynamics. Yet it's readily apparent that the factors focal in these different research projects aren't related to one another as parts and wholes, mechanisms and their components, or realization/implementation, the primary candidates for articulating levels. Bickle claimed that a social factor like poverty must be "transduced" into biochemical mechanisms, but even if relevant biochemical pathways are identified for poverty, these are not parts of poverty nor do they implement poverty. Instead, poverty may cause certain biochemical happenings. Poverty is also related causally (if at all) to personality and cognitive traits such as antisocial personality disorder and rejection sensitivity; these certainly do not relate as partswholes or realization/implementation. Some social and environmental factors might affect 
personality, cognitive features, and embodied perceptual characteristics, and vice versa, but again, this connection is causal influence, not a compositional or determining relationship.

We suspect that this point about the relationship among cognitive science insights into criminal behavior also applies to most other investigations in cognitive science. Perhaps sometimes, different factors targeted in different investigations are related compositionally, mechanistically, or in some other way that supports referring to them as different levels of explanation. But, by and large, "levels" seems misleading in this context. To be clear, we grant the existence of part-whole and realization/implementation relationships, and this discussion is not meant to convince readers that there are no levels of organization. Rather, our point is that the entities and properties focal to different cognitive science explanations are not usually related to one another in any of the primary ways used to articulate levels.

Perhaps the strongest intuitions in favor of a level relationship among cognitive science approaches relate to the role of neural processes, which are thought to implement cognitive functions. Yet personality and cognitive traits, traditionally seen as the level immediately above the brain, have not been shown to relate in this way to patterns of neural activity. Our claim is not that cognition is somehow spookily accomplished without neurological processes. Rather, the idea is that neuroscientists choose to focus on certain neural phenomena based on promising neuroscientific questions, cognitive psychologists choose to focus on certain cognitive traits based on promising questions about cognition, and so on, and there's no reason to expect that these phenomena focal to various approaches to explanation will be related in any of the ways anticipated for levels. This point is even more obvious for situational and social factors. If, for example, interpersonal dynamics help account for social coordination and cohesion (Marsh et al., 2009), then the neural characteristics of individuals may be enabling conditions for social coordination and cohesion, but they cannot by themselves implement interpersonal dynamics. ${ }^{2}$

\footnotetext{
${ }^{2}$ See Potochnik (2010) for a more extended argument for this point about levels of explanation in general.
} 
The entities and properties focal to different cognitive science explanations, including the molecular, neurological, representational/computational, and social/cultural factors often invoked in discussions of levels of explanation, are thus not generally related by part-whole composition, mechanistic composition, or realization/implementation. Rather, these are usually best viewed as causally related to one another. Sometimes one investigation may focus on some part of an entity or mechanism focal in another investigation, such as (a) investigation of how the MAOA enzyme influences dopamine levels and (b) investigation of dopamine levels across the brain. But this is uncommon, and when it does occur, the investigation of the part tends to regard only one limited component of a broader phenomenon and situates it in the context of different research interests (such as, in this case, the effects of the MAOA gene.)

Instead of appealing to part-whole, mechanism-component, or realization/implementation relationships, one may try to distinguish levels of explanation based on different levels of analysis, such as implementation, algorithmic, and computational levels, with neural events and structure belonging to the implementation level. But this is no more promising. The presumed levels of explanation in cognitive science do not by and large correspond to this articulation of levels of analysis. For example, social coordination and organism-environment relations are not implemented in the brain, or at least not entirely; the environmental and social structure also participate in the implementation. Further, the idea that different cognitive science approaches provide different levels of analysis presumes that these approaches are ordered in their degree of abstraction or generality, with neural explanations more specific than representational/computational explanations, which are in turn more specific than social/cultural explanations. This is not the case. Neuroscience offers some quite general insights into neural and brain region activity, as well as highly specific treatments of neural processes involved in certain cognitive phenomena. The findings of cognitive psychology and ecological approaches can similarly be highly specific or quite general, depending on the targeted phenomenon. Research into the dynamics of group coordination can be quite general, 
or highly specific, like the finding that children attribute less interest in play to non-handicapped children interacting with mildly handicapped playmates (Van Acker and Valenti, 1989).

Some cognitive scientists emphasize scales rather than levels. We endorse this shift, as comparisons in spatial scale or temporal scale do not have some of the problematic implications plaguing levels (Potochnik and McGill, 2012). But approaches to cognitive science explanation are not in general ordered by spatial or temporal scale any more than they are by level of organization. There is a spatial scaling from brain to organism to group, but there is not a similar temporal scaling. For example, neural reuse (Anderson, 2014, 2016) is a principle of functional organization in the brain that occurs phylogenetically and ontogenetically; explanations of behavior in terms of neural reuse thus cite entities at a small spatial scale but at a very broad range of timescales. And then, the spatial scaling of brain-organism-group doesn't continue across other phenomena investigated in the cognitive sciences. Ecological psychologists often describe their research as focusing on the "organism-environment level [of analysis]" and the "ecological scale" (e.g. Wagman and Miller, 2003). But organism-environment relations occur across a number of different spatial scales, some of which are much smaller than the human brain.

We have suggested that the entities and properties focal to different approaches to cognitive science explanations, including molecular, neurological, representational/computational, and social/cultural, are not related in the characteristic ways anticipated for levels relationships—by part-whole, realization/implementation, or degree of abstraction. Rather, they most often relate causally, as different influences on complex cognitive phenomena. Nor is there a characteristic spatial or temporal scale relationship for the different approaches to cognitive science explanations. Thus, levels (or scales) of explanation is simply not the right way to pose the question about the explanatory value of different approaches in the cognitive sciences, no matter what your view is regarding that value. Thinking of these 
approaches to explanation as levels suggests relationships among them that by and large fail to obtain.

\section{Explaining with Patterns}

In Section 2, we argued that the standard positions on the question of levels of explanation in cognitive science share a mistaken presupposition about how the candidate explanations relate to one another. By and large, properties and entities featured in these explanations are not related to one another in any of the ways that have been used to articulate levels. Rather, they tend to be causally related. Different approaches to explanation feature different causal influences on a target phenomenon, at least some of which may causally influence one another. We thus urge a reframing of the questions of which approaches explain cognitive phenomena and how these explanations relate to one another.

But the basic questions still remain: which approaches explain cognitive phenomena, and how do these explanations relate to one another? Given what we have said about different approaches featuring different causal influences, one may think we should urge explanatory integration; we might need all of these different approaches working together to fully explain cognitive phenomena. But, while it is certainly true that evidence-sharing and other forms of coordination are valuable when grappling with such complex phenomena, we think the lesson to draw for explanation is not integration but pluralism.

One of the sources of differences among cognitive science research projects is in their aim: what phenomena they address, what methods they bring to bear, and toward what specific goal. This hearkens back to Dale et al.'s (2009) mention of the "diverse explanatory goals of human beings," as quoted above. Differences in aim occasion focus on different features of cognitive phenomena. Different research projects in cognitive science thus give insight into different cognitive phenomena, and even into different features of the same cognitive phenomena. Pursuing integrated explanations that incorporate other features beyond what is 
focal is time- and resource-intensive, and this is unnecessary to generate satisfactory explanations of many cognitive phenomena.

So, we agree with those who advocate explanatory pluralism in the cognitive sciences. But our version of pluralism does not emphasize different, independent "levels" of explanation. Rather, on the view we favor-pluralism of explanatory style-different, independent explanations in cognitive science provide cross-cutting accounts, sometimes even of the same phenomena, that capture different causal patterns. The idea that causes are central to scientific explanation is widely held. Some philosophers have also suggested that laws or other broad regularities are explanatory (Hempel, 1948; Friedman, 1974; Strevens, 2008), and some cognitive science research has backed this up (Lombrozo, 2010). The idea that causal patterns are explanatory is simply the idea that information about (1) causal dependence and (2) the scope of that dependence is explanatory. This is precisely the information needed to predict and intervene on our world: information about what factors influence a phenomenon, and in what circumstances. For a general argument that causal patterns are central to scientific explanation, see Potochnik (2017).

The pluralism of explanatory styles we propose is related to the notion of "scientific styles," or the material, technical, conceptual, linguistic and social practices that vary across research projects (Hacking, 1992, 1994; Feyerabend, 1996). Scientific styles are thought to shape not only how knowledge is pursued but also what counts as knowledge and what that knowledge is of. We have something similar in mind here, but two differences are worth noting. First, some discussions of scientific styles emphasize that different fields adopt different styles (e.g., Bueno 2012). Here we emphasize the diversity of styles even within the field of cognitive science. Second, our pluralism is specifically about styles of explanation rather than styles of

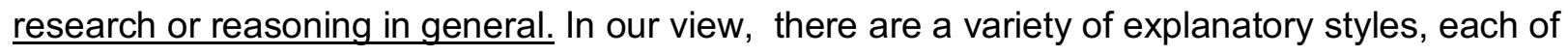
which captures certain causal patterns in cognitive phenomena. 
Let's return again to the example of criminal behavior. Forensic neuroimaging looks for neural biomarkers of aggression and impulsivity_aiming to find patterns in how certain neural features relate to propensity toward criminal behavior. Personality psychology in turn may target patterns in the role of antisocial personality disorder. Each of these investigations operationalizes the phenomenon of criminal behavior in ways convenient for its own purposes, investigates the role of factors of interest given their broader research aims, and connects these factors to different ranges of related phenomena. After all, neither research into neural patterns related to aggression and impulsivity nor research into antisocial personality disorder is confined to a focus on criminal behavior. (See Longino, 2013, for an extended case study of how these and other features vary among investigations of human aggression.) Thus, in these examples and others across cognitive science, a specific explanatory style is adopted in light of the variety of causal pattern deemed to be of interest. This results in a pluralism of multiple, independent explanations, even of a single phenomenon targeted in more than one style of investigation.

There are three important differences between our proposed pluralism of explanatory style and a pluralism about explanatory "level." First, as suggested just above, the various explanations of a given phenomenon are recognized to be cross-cutting; they are not anticipated to bear a neat relationship to one another, such as implementation/computation or components and subcomponents. Second, because of this, it is more readily apparent that each style of explanation neglects some important causal factors, factors that are relevant to bringing about the phenomenon of interest but not relevant to the specific causal pattern that is focal. On this view, which style of explanation is warranted depends on the specifics of the research program, including researchers' interests, methods, and other idiosyncrasies that influence their pursuit of explanation. Third, pluralism of explanatory styles and its emphasis on causal patterns makes clear the costs of explanatory integration. Additional causal information is not merely unimportant; on this view, it is contraindicated. It's not just that integrated explanations are timeand resource-expensive, as we noted above. Integrated explanations also are more limited than 
explanations targeted at a causal pattern of interest, since the additional causal information occludes the causal pattern of immediate interest with details that may be causally important but are incidental to the immediate research aims (Potochnik 2017). These differences are summarized in Table 1.

\section{[Insert Table 1]}

In summary, in our view, there is a variety of cross-cutting styles of cognitive science explanations, capturing different causal patterns and responding to different research interests. Neuroscience rightly focuses on experimentally detecting patterns in neural activation and brain area-behavior relationships, whereas these can be wholly tangential to cognitive psychology's focus on computationally modelling behavior in terms of information processing. Embodied and ecological approaches, in turn, frame perception-action cycles as features of entire animalenvironment systems, and accordingly (rightly) focus on interaction dynamical patterns to the exclusion of neural and (sub)personal dynamical patterns. Which explanatory style is warranted depends on the cognitive phenomenon under investigation, but this also depends on the interests and cognitive needs of those seeking the explanation, as those help determine which causal pattern is enlightening.

\section{Different Explanatory Styles in the Cognitive Sciences}

Reframing the question of cognitive science explanation as a debate not about discrete levels but about cross-cutting patterns is not empty philosophical debate about words. Rather, this reframing changes how we should answer the basic questions identified at the outset of this paper of which cognitive science approaches explain and how these explanations relate to one another. On our view of pluralism of explanatory styles, different approaches offer independent explanations, even of the same phenomena, in terms of the distinct causal patterns they focus on. The value of these explanations partly derives from their identification of broad patterns in causal influence; this distinguishes our emphasis on causal patterns from an emphasis on mere 
causal information or causal mechanism. This places the researchers who seek explanation centerstage: which kinds of potential causes are of immediate interest to them is crucial to determining which kind of explanatory style is called for.

This may make it seem like we're downplaying the differences between cognitive science explanations, as if which is warranted is merely a matter of taste. That there is a diversity of interests and of cognitive needs at play in cognitive science may seem insufficient to motivate pluralism, especially when compared to pluralism motivated by the belief in different levels. But we are not suggesting that differences among cognitive science approaches are simply a matter of preference. Rather, a successful explanatory style is identified when the right research question is posed and a pattern is uncovered in the causal action of some factor of interest. This is, we submit, what it takes to explain. An individual scientist's preference is not sufficient to justify the choice of explanatory style for a given research project. Indeed, it doesn't even seem right to think in terms of choice. Training within a given scientific field ultimately shapes the practitioners' cognitive needs and interests, delimiting in specific ways the range of explanatory styles available to anyone working within that field-determining which options are "live" and which are "dead," to use James's (1896) terms.

On the pluralism of explanatory styles that we urge, and in contrast to explanatory integration, it is not desirable for explanations to include as much causal information as possible relevant to the phenomenon to be explained. Rather, only information about causes of interest, together with the scope of their influence-the focal causal pattern-is explanatory. It is no shortcoming for an explanation of a cognitive phenomenon to omit information about causes of that phenomenon that are incidental to the focal causal pattern. This accords with research practices in the cognitive sciences. Neuroscience explanations regularly omit reference to environmental influences, cognitive psychology explanations regularly omit reference to noncognitive influences, and embodied and extended explanations regularly omit reference to neurological details. Each explanatory style benefits from these kinds of exclusions. 
There are, though, two ways in which causal pattern explanations can go wrong. There are two requirements for successful causal pattern explanations: (1) the causal pattern depicted must be responsible for the phenomenon to be explained, and (2) the causal pattern depicted must be of interest to the research program occasioning the explanation. Two forms of criticism of causal pattern explanations are thus possible. First, an explanation is not successful if the causal pattern it depicts fails to obtain. "Just so" stories are not explanations. This sharpens the questions that should be asked about any given approach in the cognitive sciences. Consider, for example, embodied, ecological, and dynamical approaches, since the status of these are relatively contentious. If we are right about a pluralism of explanatory styles, it is not sufficient to criticize these approaches for not including neurological details, nor for focusing on different kinds of causal influences than other cognitive science approaches. One should instead ask whether patterns in fact exist in how ecological factors and embodiment influence cognitive phenomena. (In many cases, the answer is yes.)

The second form of criticism regards not causal facts but research interests. Even when patterns exist in the causal influence of some factor, it may be dubious as a research focus, or it may be fairly criticized as less important than other causal patterns that aren't receiving similar focus. Amos et al. (2010) find an association between a certain genetic variant and the propensity to initiate smoking. But their findings, even if legitimate, are surely on balance unimportant to research into smoking. Many other research questions are better positioned to help us understand and intervene on this public health concern.

From the viewpoint of pluralism in explanatory styles, any approach in cognitive science can offer explanations if it successfully identifies a causal pattern of interest that accounts for the phenomenon under investigation. Different approaches are thus not offering competing explanations, but compatible, independent explanations of different phenomena, or even of the same phenomena but focused on different causal patterns. None of what we have said undermines the importance of interdisciplinary exchange. The cognitive sciences address a 
range of related phenomena; sharing data and methods is undoubtedly valuable. Indeed, developing integrated explanations across disciplines can be a worthy project. But, in our view, this is just one kind of explanatory aim among many.

Our account of cross-cutting patterns and the resulting pluralism of explanatory styles reframes the debate about cognitive science explanation: the question is not which levels are explanatory, but which explanatory style is called for given the potential causal pattern of interest. This clarifies the questions that should be asked to ascertain whether any given field explains cognitive phenomena, and this changes our expectations for how different cognitive science explanations relate to one another. Thus, in our view, levels-thinking shouldn't influence how disciplines within cognitive science view their respective explanatory projects. Those projects are not neatly aligned, and none---even explanatory integration---takes priority over the others. Extra causal detail obscures the very patterns that are explanatory for a given research focus. There's much to be gained from recognizing the pluralism of explanatory styles across the cognitive sciences.

\section{Acknowledgments}

We thank Matteo Colombo and Markus Knauff for inviting our participation in this interesting issue and four anonymous reviewers for helpful comments.

\section{References}

Aharoni, E., Vincent, G. M., Harenski, C. L., Calhoun, V. D., Sinnott-Armstrong, W., Gazzaniga, M. S., \& Kiehl, K. A. (2013). Neuroprediction of future rearrest. Proceedings of the National Academy of Sciences, 201219302.

Anderson, M. L. (2014). After phrenology (Vol. 547). Cambridge, MA: MIT Press.

Anderson, M. L. (2016). Précis of after phrenology: neural reuse and the interactive brain. Behavioral and Brain Sciences, 39.

Amos, C. I., Spitz, M. R., \& Cinciripini, P. (2010). Chipping away at the genetics of smoking behavior. Nature genetics, 42(5), 366. 
Barrett, L., Henzi, S. P., \& Lusseau, D. (2012). Taking sociality seriously: the structure of multidimensional social networks as a source of information for individuals. Philosophical Transactions of the Royal Society of London B: Biological Sciences, 367(1599), 2108-2118.

Bartolo, T., Peled, M., \& Maretti, M. M. (2009). Social-cognitive processes related to risk for aggression in adolescents. Court Review, 46, 44.

Bennett, S., Farrington, D. P., \& Huesmann, L. R. (2005). Explaining gender differences in crime and violence: The importance of social cognitive skills. Aggression and violent behavior, 10(3), 263-288.

Bickle, John. 2003. Philosophy and neuroscience: A ruthlessly reductive account. Springer.

Bickle, John. "Laser lights and designer drugs: New techniques for descending levels of mechanisms 'in a single bound"', TopiCS (forthcoming).

Brown, B. B., \& Altman, I. (1983). Territoriality, defensible space and residential burglary: An environmental analysis. Journal of Environmental Psychology, 3(3), 203-220.

Bueno, O. (2012). Styles of reasoning: A pluralist view. Studies in History and Philosophy of Science Part A, 43(4), 657-665.

Bufkin, J. L., \& Luttrell, V. R. (2005). Neuroimaging studies of aggressive and violent behavior: current findings and implications for criminology and criminal justice. Trauma, Violence, \& Abuse, 6(2), 176-191.

Clarke, R. V. (1980). Situational crime prevention: Theory and practice. British Journal of Criminology, 20, 136.

Coleman, P. T., Vallacher, R. R., Nowak, A., \& Bui-Wrzosinska, L. (2007). Intractable conflict as an attractor: A dynamical systems approach to conflict escalation and intractability. American Behavioral Scientist, 50(11), 1454-1475.

Craver, Carl F. (2007). Explaining the Brain: Mechanisms and the Mosaic Unity of Neuroscience. Oxford UP.

Dale, R., Dietrich, E., \& Chemero, A. (2009). Explanatory pluralism in cognitive science. Cognitive science, 33(5), 739-742.

Donovan, G. H., \& Prestemon, J. P. (2012). The effect of trees on crime in Portland, Oregon. Environment and Behavior, 44(1), 3-30.

Feyerabend, P. (1996). Theoreticians, artists and artisans. Leonardo, 29(1), 23-28.

Friedman, M. (1974). Explanation and scientific understanding. The Journal of Philosophy, 71(1), 5-19.

Gallagher, S., Janz, B., Reinerman, L., Trempler, J., \& Bockelman, P. (2015). A Neurophenomenology of Awe and Wonder: Towards a Non-Reductionist Cognitive Science. Springer. 
Hacking, I. (1992). 'Style' for historians and philosophers. Studies in History and Philosophy of Science Part A, 23(1), 1-20.

Hacking, I. (1994). Styles of scientific thinking or reasoning: A new analytical tool for historians and philosophers of the sciences. Trends in the Historiography of Science (pp. 31-48). Springer, Dordrecht.

Hempel, C. G., \& Oppenheim, P. (1948). Studies in the Logic of Explanation. Philosophy of science, 15(2), 135-175.

James, W. (1896). The will to believe: And other essays in popular philosophy. Longmans, Green and Co.

Kandel, E. R., Schwartz, J. H., Jessell, T. M., Siegelbaum, S. A., and Hudspeth, A. J. (2013) Principles of Neural Science. McGraw-Hill.

Kitcher, P. (1984). 1953 and all that. A tale of two sciences. The Philosophical Review, 93(3), 335-373.

Krakauer, J. W., Ghazanfar, A. A., Gomez-Marin, A., Maclver, M. A., \& Poeppel, D. (2017). Neuroscience needs behavior: correcting a reductionist bias. Neuron, 93(3), 480-490.

Kuo, F. E., \& Sullivan, W. C. (2001). Environment and crime in the inner city: Does vegetation reduce crime? Environment and behavior, 33(3), 343-367.

Lombrozo, T. (2010). Causal-explanatory pluralism: How intentions, functions, and mechanisms influence causal ascriptions. Cognitive Psychology, 61(4), 303-332.

Longino, H. E. (2013). Studying human behavior: how scientists investigate aggression and sexuality. University of Chicago Press.

Marr, D. (1982). Vision: A computational investigation into the human representation and processing of visual information. MIT Press.

Marsh, K. L., Richardson, M. J., \& Schmidt, R. C. (2009). Social connection through joint action and interpersonal coordination. Topics in Cognitive Science, 1(2), 320-339.

McArthur, L. Z., \& Baron, R. M. (1983). Toward an ecological theory of social perception. Psychological review, 90(3), 215.

Miłkowski, M., Clowes, R.W., Rucińska, Z., Przegalińska, A., Zawidzki, T., Gies, A., Krueger, J., McGann, M., Afeltowicz, Ł., Wachowski, W.M. and Stjernberg (2018). From Wide Cognition to Mechanisms: A Silent Revolution. Frontiers in psychology, 9, 2393.

Perkins, D. D., Wandersman, A., Rich, R. C., \& Taylor, R. B. (1993). The physical environment of street crime: Defensible space, territoriality and incivilities. Journal of environmental psychology, 13(1), 29-49.

Potochnik, A. (2010). Levels of explanation reconceived. Philosophy of science, 77(1), 59-72. 
Potochnik, A., \& McGill, B. (2012). The limitations of hierarchical organization. Philosophy of Science, 79(1), 120-140.

Potochnik, A. (2017). Idealization and the Aims of Science. University of Chicago Press.

Richardson, R. C. (2009). Multiple realization and methodological pluralism. Synthese, 167(3), 473-492.

Riser, R. E., \& Kosson, D. S. (2013). Criminal behavior and cognitive processing in male offenders with antisocial personality disorder with and without comorbid psychopathy. Personality Disorders: Theory, Research, and Treatment, 4(4), 332.

Strevens, M. (2008). Depth: An account of scientific explanation. Harvard University Press.

Tesser, A., \& Achee, J. (1994). Aggression, love, conformity, and other social psychological catastrophes. In R. R. Vallacher \& A. Nowak (Eds.), Dynamical systems in social psychology. Academic Press.

Thagard, P. (2005). Mind: Introduction to Cognitive Science. MIT Press.

Topalli, V. (2005). Criminal expertise and offender decision-making: An experimental analysis of how offenders and non-offenders differentially perceive social stimuli. The British Journal of Criminology, 45(3), 269-295.

Topalli, V., Jacques, S., \& Wright, R. (2015). "It takes skills to take a car": Perceptual and procedural expertise in carjacking. Aggression and Violent Behavior, 20, 19-25.

Van Acker, R., \& Valenti, S. S. (1989). Perception of social affordances by children with mild handicapping conditions: Implications for social skills research and training. Ecological psychology, 1(4), 383-405.

Renaud, P., Chartier, S., Rouleau, J.L., Proulx, J., Goyette, M., Trottier, D., Fedoroff, P., Bradford, J.P., Dassylva, B. and Bouchard, S., (2013). Using immersive virtual reality and ecological psychology to probe into child molesters' phenomenology. Journal of Sexual Aggression, 19(1), 102-120.

Wagman, J. B., \& Miller, D. B. (2003). Nested reciprocities: The organism-environment system in perception-action and development. Developmental Psychobiology: The Journal of the International Society for Developmental Psychobiology, 42(4), 317-334. 


\section{Pluralism of explanatory level}

Pluralism of explanatory style

1. Explanations anticipated to be ordered by, Explanations recognized as cross-cutting

e.g., part-whole, implementation, or scale

2. Level of explanation determines what Research interests determine which causes kind of information is explanatory

to include and which to exclude
3. Extra causal information unimportant
Extra causal information detrimental to depicting causal pattern

Table 1: The features of our proposed pluralism of explanatory style contrasted with an explanatory pluralism developed in terms of levels of explanation. 